\title{
氧空位依赖的氯氧化铋光催化反应
}

杨金龙

中国科学技术大学化学与材料科学学院, 合肥 230026

\section{Oxygen Vacancy-Dependent Photocatalysis of Bismuth Oxychloride}

\section{YANG Jinlong}

School of Chemistry and Materials Science, University of Science and Technology of China, Hefei 230026, P. R. China.

Email: jlyang@ustc.edu.cn

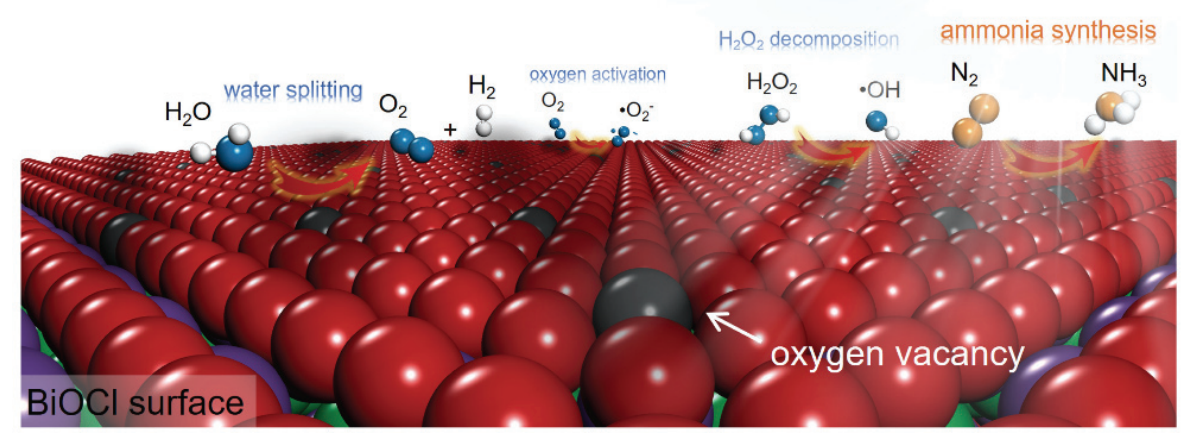

常见的小分子 $\left(\mathrm{O}_{2}, \mathrm{H}_{2} \mathrm{O}, \mathrm{H}_{2} \mathrm{O}_{2}, \mathrm{~N}_{2}\right)$ 在 $\mathrm{BiOCl}$ 氧空位上的吸附、活化和光催化反应

随着现代材料表征技术与理论模拟计算的协 同发展, 人们逐渐认识到对于实际的催化剂, 其表 面少量缺陷相比于其体相结构和化学组成起着更 重要的作用 1 。鉴于此, 深刻地理解表面氧空位与 光催化中动力学、热力学以及反应路径之间的关 系非常重要。其中氧空位是最常见并且研究最多 的一种阴离子缺陷 ${ }^{2}$ 。最近, 华中师范大学化学学 院张礼知教授研究团队结合氧空位依赖的光催化 表面化学的研究现状及其团队在该领域的最新研 究进展, 在 Angewandte Chemie International Edition 上撰写了题为 “Oxygen Vacancy-Mediated Photocatalysis of BiOCl: Reactivity, Selectivity and Perspective” 的综述 ${ }^{3}$ 。

作为三元氧化物的代表物之一, $\mathrm{BiOCl}$ 具有独 特的各向异性层状结构。不同于其他半导体材料, $\mathrm{BiOCl}$ 的表面结构(如特定晶面暴露和表面氧空 位)不仅易于调控, 还呈现出新颖的晶面相关物理 化学性质和氧空位介导光催化反应过程。在分子
氧活化方面, $\mathrm{BiOCl}(001)$ 面氧空位可以通过单电子 还原路径 $\mathrm{O}_{2}+\mathrm{e}^{-} \rightarrow \cdot \mathrm{O}_{2}^{-}$活化氧分子到超氧负离 子, 并进一步通过 $\cdot \mathrm{O}_{2}^{-} \rightarrow \mathrm{O}_{2}^{2-} \rightarrow \mathrm{H}_{2} \mathrm{O}_{2} \rightarrow \cdot \mathrm{OH}$ 的 途径产生其它活性氧物种; $\mathrm{BiOCl}(010)$ 面氧空位则 通过双电子还原路径 $\mathrm{O}_{2}+2 \mathrm{H}^{+}+2 \mathrm{e}^{-} \rightarrow \mathrm{H}_{2} \mathrm{O}_{2}$ 直接活 化氧分子到双氧水。这些活性氧物种赋予了 $\mathrm{BiOCl}$ 优良的污染物氧化去除和选择性氧化能力 ${ }^{4-6}$ 。水分 子在不同晶面的 $\mathrm{BiOCl}$ 氧空位上会表现出不同地 吸附构型, 其中在 $\mathrm{BiOCl}(001)$ 面氧空位上, 水分 子以分子态吸附; 而在(010)面氧空位上水分子则 以解离态吸附 7 。由于解离态吸附的水分子克服了 水氧化中第一质子转移反应 $\left(\mathrm{H}_{2} \mathrm{O}+\mathrm{h}^{+} \rightarrow \cdot \cdot \mathrm{OH}+\right.$ $\mathrm{H}^{+}$) 的能垒, 进而表现出较高的活化状态, 其相比 于分子态吸附的水分子更容易在热力学和动力学 上捕获空穴。氧空位还被第一次证实为双氧水解 离吸附的活性位点, 能直接诱发双氧水中 $\mathrm{O}-\mathrm{O}$ 键 的异裂, 产生一个占据在氧空位处的 $\mathrm{OH}^{-}$以及一 个羟基自由基, 而羟基自由基的存在形式与氧空 
位的结构息息相关 ${ }^{8}$ 。 $\mathrm{BiOCl}(001)$ 面上的氧空位还 能诱导产生游离的羟基自由基, 而(010)面上的氧 空位则选择性地产生表面羟基自由基。不同存在 形式的羟基自由基表现出不同的有机污染物去除 特性。由于其富电子的特性, 氧空位还可以有效地 活化氮气中 $\mathrm{N} \equiv \mathrm{N}$ 三键, 从而增大在其动力学上被 还原的速率 ${ }^{9}$ 。不仅如此, 在 $\mathrm{BiOCl}(001)$ 面产生的 氧空位上, 氮分子是以 “terminal end-on” 模式吸 附, 并以 “asymmetric distal, $\mathrm{N}_{2} \rightarrow \mathrm{N}-\mathrm{NH}_{3} \rightarrow$ $2 \mathrm{NH}_{3}$ ” 的路径被固定, 产生 $\mathrm{NH}_{3}$ 为主要产物; 而 在(010)面的氧空位上, 氮分子则是以 “ side-on bridging” 模式吸附, 并且表现出更高的活化状 态, 以“symmetric alternating, $\mathrm{N}_{2} \rightarrow \mathrm{N}_{2} \mathrm{H}_{2} \rightarrow \mathrm{N}_{2} \mathrm{H}_{4}$ ” 的路径被固定，产生 $\mathrm{N}_{2} \mathrm{H}_{4}$ 为重要的中间产物 ${ }^{10}$ 。 这些研究结果从表面分子层面阐述了氧空位 与光催化反应的一些构效关系, 巩固并加深了半 导体表面光催化的基本理论, 为高效光催化剂的 设计提供了新思路。

\section{References}

(1) Sun, Y.; Gao, S.; Lei, F.; Xie, Y. Chem. Soc. Rev. 2015, 44, 623. doi: $10.1039 / \mathrm{C} 4 \mathrm{CS} 00236 \mathrm{~A}$

(2) Nowotny, J.; Alim, M. A.; Bak, T.; Idris, M. A.; Ionescu, M.; Prince, K.; Sahdan, M. Z.; Sopian, K.; Mat Teridi, M. A.; Sigmund, W. Chem. Soc. Rev. 2015, 44, 8424. doi: 10.1039/C4CS00469H

(3) Li, H.; Li, J.; Ai, Z. H.; Jia, F. L.; Zhang, L. Z. Angew. Chem. Int. Ed. 2017, doi: 10.1002/anie.201705628

(4) Zhao, K.; Zhang, L. Z.; Wang, J. J.; Li, Q. X.; He, W. W.; Yin, J. J. J. Am. Chem. Soc. 2013, 135, 15750. doi: 10.1021/ja4092903

(5) Li, H.; Shi, J. G.; Zhao, K.; Zhang, L. Z. Nanoscale 2014, 6, 14168. doi: 10.1039/C4NR04810E

(6) Li, H.; Qin, F.; Yang, Z. P.; Cui, X. M.; Wang, J. F.; Zhang, L. Z. J. Am. Chem. Soc. 2017, 139, 3513. doi: 10.1021/jacs.6b12850

(7) Li, H.; Shang, J.; Zhu, H. J.; Yang, Z. P.; Ai, Z. H.; Zhang, L. Z. ACS Catal. 2016, 6, 8276. doi: 10.1021/acscatal.6b02613.

(8) Li, H.; Shang, J.; Yang, Z. P.; Shen, W. J.; Ai, Z. H.; Zhang, L. Z. Environ. Sci. Technol. 2017, 51, 5685. doi: 0.1021/acs.est.7b00040

(9) Li, H.; Shang, J.; Ai, Z. H.; Zhang, L. Z. J. Am. Chem. Soc. 2015, 137, 6393. doi: $10.1021 /$ jacs.5b03105

(10) Li, H.; Shang, J.; Shi, J. G.; Zhao, K.; Zhang, L. Z. Nanoscale 2016, 8, 1986. doi: 10.1039/C5NR07380D 